\title{
An Overlapping Phenotype of Osteogenesis Imperfecta and Ehlers-Danlos Syndrome Due to a Heterozygous Mutation in COL1A1 and Biallelic Missense Variants in TNXB Identified by Whole Exome Sequencing
}

\author{
Luisa Mackenroth, ${ }^{1,2}$ Björn Fischer-Zirnsak, ${ }^{1,3}$ Johannes Egerer, ${ }^{1}$ Jochen Hecht, ${ }^{1}$ \\ Tilmann Kallinich, ${ }^{4}$ Werner Stenzel, ${ }^{5}$ Birgit Spors, ${ }^{6}$ Arpad von Moers, ${ }^{7}$ Stefan Mundlos, ${ }^{1,3}$ \\ Uwe Kornak, ${ }^{1,3}$ Kerstin Gerhold, ${ }^{4}$ and Denise Horn ${ }^{1 *}$ \\ ${ }^{1}$ Institute of Medical and Human Genetics, Charité-Universitätsmedizin Berlin, Berlin, Germany \\ ${ }^{2}$ Faculty of Medicine Carl Gustav Carus, Institute for Clinical Genetics, Dresden, Germany \\ ${ }^{3}$ Max-Planck-Institut für Molekulare Genetik, Berlin, Germany \\ ${ }^{4}$ Department of Pediatric Pneumology and Immunology, Charité-Universitätsmedizin Berlin, Berlin, Germany \\ ${ }^{5}$ Institute for Neuropathology, Charité, Universitätsmedizin Berlin, Berlin, Germany \\ ${ }^{6}$ Department of Radiology, Charité, Universitätsmedizin Berlin, Berlin, Germany \\ 'Children's Hospital, DRK-Kliniken Westend, Berlin, Germany
}

Manuscript Received: 27 April 2015; Manuscript Accepted: 21 December 2015

Osteogenesis imperfecta (OI) and Ehlers-Danlos syndrome (EDS) are variable genetic disorders that overlap in different ways [Cole 1993; Grahame 1999]. Here, we describe a boy presenting with severe muscular hypotonia, multiple fractures, and joint hyperflexibility, features that are compatible with mild OI and hypermobility type EDS, respectively. By whole exome sequencing, we identified both a COL1A1 mutation (c.4006-1G $>$ A) inherited from the patient's mildly affected mother and biallelic missense variants in TNXB (p.Val1213Ile, p.Gly2592Ser). Analysis of cDNA showed that the COL1A1 splice site mutation led to intron retention causing a frameshift (p.Phe1336Valfs*72). Type 1 collagen secretion by the patient's skin fibroblasts was reduced. Immunostaining of a muscle biopsy obtained from the patient revealed a clear reduction of tenascin- $X$ in the extracellular matrix compared to a healthy control. These findings imply that the combination of the COL1A1 mutation with the TNXB variants might cause the patient's unique phenotype. () 2016 Wiley Periodicals, Inc.

Key words: osteogenesis imperfecta; Ehlers-Danlos syndrome; COL1A1; TNXB; whole exome sequencing

\section{INTRODUCTION}

To date, a molecular diagnosis can be made for about half of the patients with suspected genetic diseases. Especially patients

\section{How to Cite this Article:}

Mackenroth L, Fischer-Zirnsak B, Egerer J, Hecht J, Kallinich T, Stenzel W, Spors B, von Moers A, Mundlos S, Kornak U, Gerhold K, Horn D. 2016. An overlapping phenotype of Osteogenesis imperfecta and Ehlers-Danlos syndrome due to a heterozygous mutation in COL1A1 and biallelic missense variants in TNXB identified by whole exome sequencing. Am J Med Genet Part A 170A:1080-1085.

presenting with non-characteristic phenotypes are difficult to classify. Connective and supportive tissue disorders can be a challenge for clinicians due to the many overlapping phenotypes.

Conflict of interest: None.

${ }^{*}$ Correspondence to:

Denise Horn, Institute of Medical and Human Genetics, CharitéUniversitätsmedizin Berlin, Augustenburger Platz 1, 13353 Berlin, Germany.

E-mail: denise.horn@charite.de Article first published online in Wiley Online Library (wileyonlinelibrary.com): 22 January 2016

DOI 10.1002/ajmg.a.37547 
Osteogenesis imperfecta (OI) is one of these clinically and genetically heterogeneous disorders resulting in osteopenia and bone fragility. According to the most recent classifications, at least 14 different types of OI can be distinguished with a clinical spectrum ranging from perinatal lethality (type 2) to milder forms presenting as a premature form of osteoporosis (type 1) [Marini and Blissett, 2013; Van Dijk and Sillence, 2014]. However, interand even intra-familial variability is common [Shapiro et al., 2013].

Most types of $\mathrm{OI}$ are due to mutations in the genes encoding the pro- $\alpha 1$ and pro- $\alpha 2$ chain of type I collagen, COL1A1 and COL1A2. Most of these mutations are considered as private, i.e., they are only found in a single family. The majority of these mutations provoke a glycine substitution in one of the 43 core exons of the 51 COL1A1 exons, which contain the GLY-X-Y triplet domain [Marini et al., 2007]. Interestingly, impaired cleavage of the N-terminal type 1 collagen propeptide, either due to mutations in type 1 collagen or the protease ADAMTS2, entails a combination of a skin phenotype with osteopenia and bone fragility [De Paepe and Malfait, 2012]. Only a small number of cases are caused by mutations in genes that play a role in the posttranslational modification of type 1 collagen including LEPRE1 (OI, type 8, OMIM \#610915) and CRTAP (OI, type 7, OMIM \#610682) leading to an autosomal recessive type of OI.

Ehlers-Danlos syndrome (EDS) has six major subtypes each with various minor forms according to the most recent classification [Beighton et al., 1998]. The clinical variability ranges from mild joint hypermobility to severe skin and joint involvement as well as life-limiting vascular fragility.

The hypermobility type EDS is considered the most common form and while chronic pain and reduced quality of life are significant features of hypermobility type EDS, life-limiting features like vascular or intestinal/uterine fragility as seen in other EDS types are uncommon. This condition is mainly inherited in an autosomal dominant manner and in some cases haploinsufficiency of the tenascin-X gene (TNXB) was shown to be the cause of the disease. A small proportion of EDS patients show homozygous or compound heterozygous mutations in TNXB leading to a distinct EDS phenotype mimicking the classic type but without delayed wound healing and atrophic scars. One main characteristic that differs from other forms of EDS is the significant generalized muscle weakness despite normal findings in standard staining of muscular biopsy [Voermans et al., 2007; Voermans et al., 2008; De Paepe and Malfait, 2012; Penisson-Besnier et al., 2013]. Tenascin-X is an extracellular matrix (ECM) protein highly expressed in skeletal muscle where it is present in the endo-, peri-, and epimysium [Voermans et al., 2008].

Here, we describe a currently 13 -year-old boy initially presenting with severe muscular hypotonia found to harbor a heterozygous mutation in COL1A1 and biallelic variants in TNXB. We discuss how the combination of these variants may cause the complex phenotype with overlapping OI/EDS features.

\section{MATERIALS AND METHODS}

\section{Patient}

DNA from the patient and his parents was obtained from peripheral blood samples according to standard procedures.
Furthermore, a skin biopsy was taken from the affected boy and his mother. Written consent for molecular studies and for publication of photographs was obtained from patient's legal representatives.

\section{Whole-Exome Sequencing}

Whole-exome sequencing (WES) was performed on DNA samples of the family trio (patient and his parents). Each DNA sample was captured using SureSelect Human All Exon Kit (Agilent Technologies, Santa Clara, CA), and sequencing of $100 \mathrm{bp}$ paired-end reads was carried out on a HiSeq 2500 (Illumina, Inc., San Diego, CA). Sequence reads were mapped to the haploid human reference genome (hg19) using Novoalign (Novocraft Technologies, Selangor, Malaysia). Single nucleotide variants (SNVs) and short insertions and deletions (indels) were called using SAMtools and resulted in a high quality exome variant set [Li, 2011; Heinrich et al., 2013]. The variant annotation on a functional level was performed using Annovar [Wang et al., 2010]. Sequence variants were filtered for a recessive mode of inheritance (homozygous and compound heterozygous) and for dominant de novo variants in GeneTalk to identify candidate variants [Kamphans and Krawitz, 2012]. All exons containing candidate variants were validated using ABI cycle sequencing. The products were separated using an ABI3730 capillary sequencer (Applied Biosystems, Life Technologies $\mathrm{GmbH}$, Darmstadt, Germany). Sequencing primers are available upon request.

\section{Cell Culture}

A skin biopsy was taken according to standard procedures from the affected boy and his mother. Fibroblasts were isolated by collagenase digestion. Isolated fibroblasts were cultivated in DMEM (HighClone) supplemented with 10\% FCS (Gibco, Thermo Fisher, Dreieich, Germany), 1\% ultraglutamine (Lonza, Verviers, Belgium), and 1\% penicillin-/streptomycin (Lonza).

\section{RNA Isolation/cDNA Synthesis}

Cells were seeded in a density of 200,000 cells per well to reach confluence directly after seeding. They were cultivated for 3 days and lysed using Trizol reagent (Invitrogen, Life Technologies GmbH, Darmstadt, Germany). Reverse transcription was performed using RevertAidTM H Minus First Strand cDNA Synthesis Kit (Fermentas, Thermo Fisher, Dreieich, Germany).

\section{Immunohistochemistry Muscle}

Seven micrometer thick cryostat sections of the patients' muscle biopsy and an age-matched control muscle biopsy from a male patient without any pathological alterations were stained by modified Gömöri trichrome, and by hematoxylin and eosine (H\&E). Immunofluorescence of TNXB was performed as previously described [Preusse et al., 2012]. Photomicrographs were taken with the Olympus BX50 microscope, the digital camera DP25, and cell* D software. 


\section{Type 1 Collagen ELISA}

Patients' and control fibroblasts were seeded at high confluence in a 24-well plate and held in standard growth medium for $48 \mathrm{hr}$ before serum content was reduced to $2 \%$. After another $48 \mathrm{hr}$, serum was further reduced to $0.5 \%$ for $72 \mathrm{hr}$ in a defined volume of growth medium. The supernatant was collected and subjected to an ELISA specific for human procollagen type $1 \mathrm{C}$-peptide PIP (Takara Bio Europe, Clontech, Saint-Germain-en-Laye, France) at a $20 \times$ dilution, following the manufacturer's protocol. Cell pellets were lysed in RIPA buffer supplemented with protein inhibitor (Roche, Basel, Switzerland) and protein content measured by BCA assay. Protein concentration was used for normalization of ELISA results.

\section{CLINICAL REPORT}

The male patient was born to non-consanguineous parents of German descent (Fig. 1). Growth parameters at birth (birth weight $3,300 \mathrm{~g}, 10 \mathrm{th}-25$ th percentile, birth length $52 \mathrm{~cm}, 25 \mathrm{th}-50$ th percentile, at 40th week of gestation) as well as occipito-frontal head circumference (OFC, $36 \mathrm{~cm}$, 50th-75th percentile) were normal. Initially, he showed mild motoric developmental delay leading to physiotherapy at the age of 14 months. He developed valgus deformity of the feet and joint hypermobility as well as severe weakness in back and neck muscles at the age of 25 months. His speech development was normal. Facio-scapulo-humeral muscle dystrophy, hereditary neuropathy Charcot-Marie-Tooth, and homozygous deletions in the SMN1 and -2 genes for types 1-3 spinal muscle atrophy were molecular genetically excluded. Normal results of histologic examination of a muscle biopsy (including electron microscopy) did not confirm the suspected diagnosis of congenital myopathy at the age of 10 years.
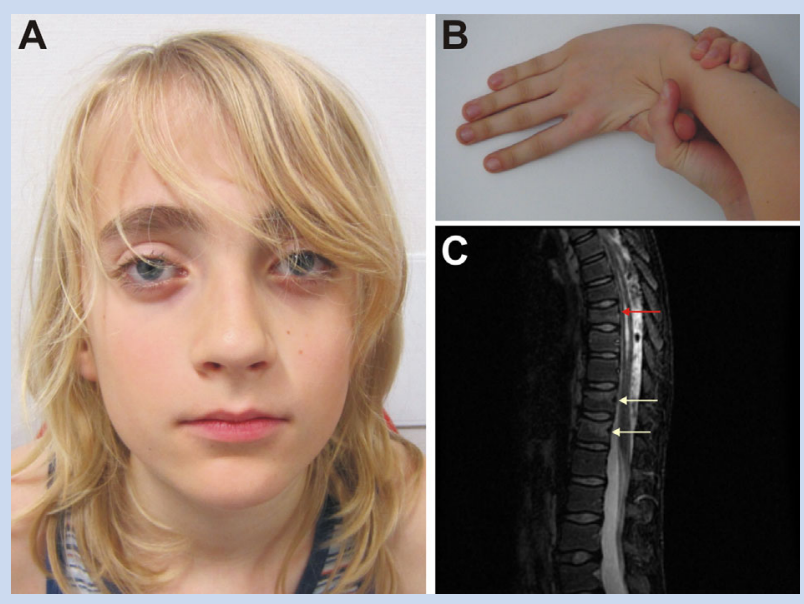

FIG. 1. Clinical presentation of index patient. (A) Mild protrusio bulbi, (B) finger joint hypermobility, (C) sagital STIR sequence: osseous edema, loss of vertebral body height with impaction of the upper and lower vertebral endplate T11 and T12 (white arrows], abnormally-shaped vertebra T8 (red arrow). [Color figure can be seen in the online version of this article, available at http://wileyonlinelibrary.com/journal/ajmga].
History of fractures started at the age of 15 months in form of a greenstick fracture of the right femur followed by bilateral wrist fractures and a fracture of one metatarsal bone at the age of 10 years. All of these five fractures occurred without adequate trauma. Due to this, bone phenotype DXA measurements were performed showing osteoporosis of the spine (Z-score -2.8).

Examination at 11 years of age revealed the following features: His height was $140 \mathrm{~cm}$ (25th percentile), weight $29 \mathrm{~kg}(<3 \mathrm{rd}$ percentile), and OFC $56 \mathrm{~cm}$ (97th percentile); he had prominent forehead, mildly protruding bulbi, muscular hypotonia, joint hypermobility of large and especially of finger joints (Beighton Score 6/9), scapulae alatae, white sclerae, and no dental abnormalities. There was no abnormality in scarring or disposition to hematomas and no hyperelastic skin. No hearing loss was present. At the age of 11 years, echocardiography gave a normal result. At the age of 12 years, an MRI study of the spine showed loss of vertebral body height with impaction of the upper and lower vertebral endplates T11 and T12, and a wedge-shaped vertebra T8.

Later results of WES led to closer clinical evaluation of the mother who was initially thought to be unaffected. We found her to present with mild joint hypermobility with a Beighton score of 5/9. Further, radiological examination based on her pain history revealed three rib fractures that occurred in adult age following inadequate trauma. DXA measurements revealed an osteopenia in her spine (T-score -2.2, Z-score -1.5). Neither cardiac symptoms nor skin abnormalities (easy bruising, laxity, etc.) were seen. Altogether, the mother appeared to have a significantly less severe phenotype than the patient. The father did not present with any abnormalities of the skin, muscles, joints, or bones.

After myocarditis in adult age, he developed mild cardiac valvular insufficiency.

\section{RESULTS OF MOLECULAR INVESTIGATIONS}

WES showed a total of 221,760 variants in the family trio. Filtering for autosomal dominant de novo variants in GeneTalk revealed 22 variants. Neither of these variants, nor any of the 29 variants determined by filtering for recessive homozygous variants were obviously associated with the patient's phenotype (Supplementary Table I). Filtering for compound heterozygous recessive variants revealed 46 variants, among them, two variants in TNXB: c.3637G $>$ A (p.Val1213Ile) and c.7774G > A (p.Gly2592Ser). Both variants are not contained in the exome variant server dataset.

With regard to multiple fractures without adequate trauma, we also filtered for variants in genes known to be associated with $\mathrm{OI}$ : COL1A1, COL1A2, CRTAP, FKBP10, IFITM5, LEPRE1, SERPINF1, SERPINH1, and PPIB. Through this, we found the novel heterozygous splice site mutation c.4006-1G > A in COL1A1 not only in the patient (detectable in 14 out of 23 reads), but also in his mother (detectable in 11 out of 14 reads). Due to the presence in mother and child, this mutation did not appear in the primary filtering for dominant de novo mutations. The exchange affects the last base pair of the splice acceptor site at the end of intron 49 . MutationTaster predicted this mutation to be disease causing. This mutation, as well as the two TNXB variants, was validated by Sanger sequencing (Fig. 2). 


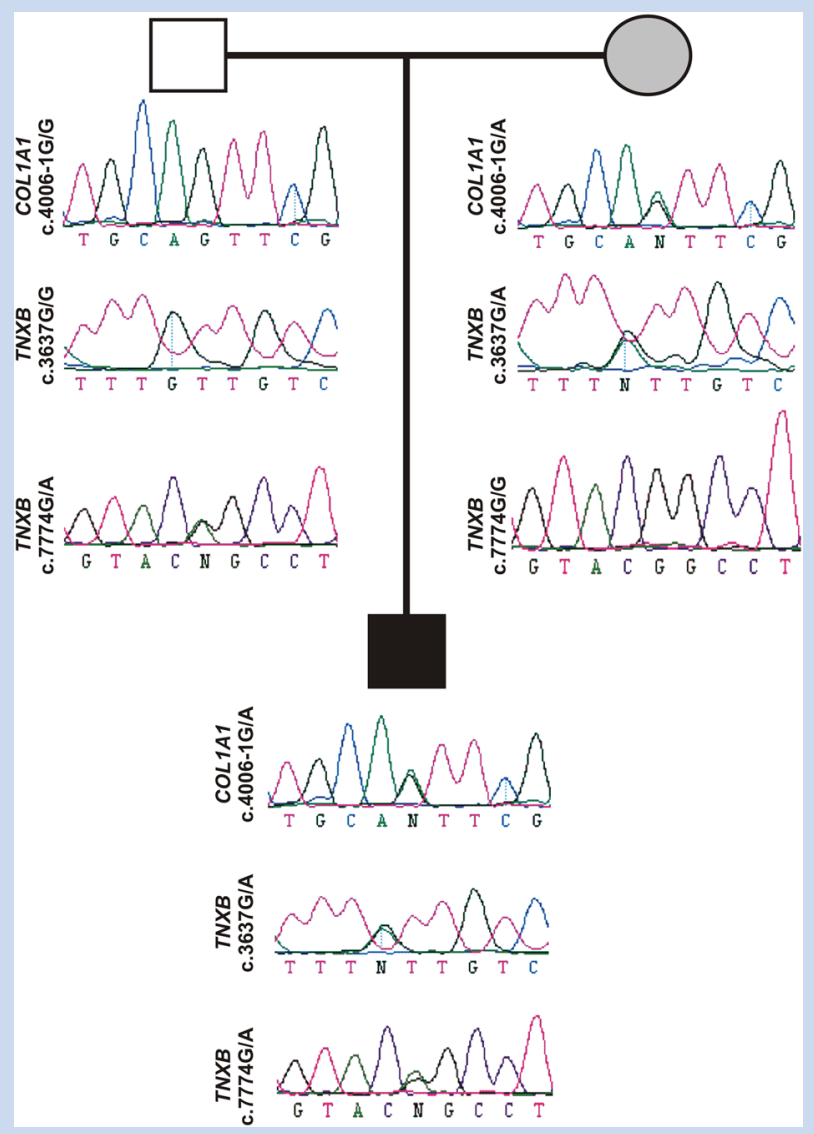

FIG. 2. Variants in TNXB and the COL1A1 mutation found in the family as validated by Sanger sequencing. The COL1A1 mutation was found in the index and his mother. As shown, compound heterozygosity of the TNXB variants was confirmed by Sanger sequencing. [Color figure can be seen in the online version of this article, available at http://wileyonlinelibrary.com/journal/ ajmga].

Further examination of the predicted splice site mutation in COL1A1 was carried out using cDNA from fibroblasts of the index and his mother. Using primers placed in intron 49 and exon 50, a 728 bp product in the cDNA from the index case and his mother was detected, but not in controls. By direct sequencing of the PCR product, we could show a retention of intron 49 in the index patient and his mother leading to a frameshift product with a premature stop codon (p.Phe1336Valfs*72) (Fig. 3A,B).

The phenotypic differences between patient and mother suggested the presence of an additional genetic factor in the index patient. This drew closer attention to the two compound heterozygous missense variants in TNXB. While the variant c.3637G > A (p.Val1213Ile) located in exon 9 was of maternal origin, the variant in exon 22 c.7774G > A (p.Gly2592Ser) was inherited from the father (Fig. 2). The paternally inherited variant was not seen in the ExAC dataset. However, the maternal variant appears twice in this dataset in a heterozygous state. Both variants were predicted by MutationTaster to be polymorphisms. PolyPhen2 ranked the paternally inherited variant as probably damaging. In addition,

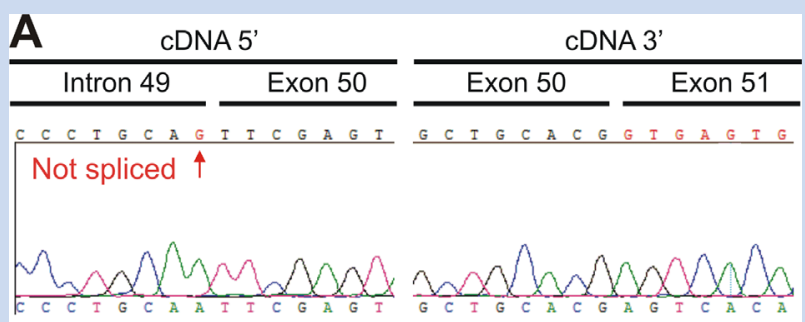

B

Wild Type

\begin{tabular}{lll|l|l|} 
c.4006-1G>A & Exon $49 \quad$ Intron 49 | Exon $50 \quad$ Exon 51
\end{tabular}

FIG. 3. Localization and effect of the COL1A1 mutation, $[A]$ the base pair exchange is located at the very last base pair of intron 49 , thus affecting the splice acceptor site and leading to preservation of intron 49 in the index patient and his mother as opposed to control cases on the right side, where intron 49 is spliced out; this leads to a premature stop codon in the preserved intron 49 (red bar). (B) Schematic overview of the splice products resulting from the two different COL1A1 alleles in the affected boy. The premature termination codon is indicated in red. [Color figure can be seen in the online version of this article, available at http://wileyonlinelibrary.com/journal/ajmga].

SIFT predicted this variant to be deleterious and MutationAssessor predicted a medium functional impact. Comparison across species showed a higher conservation for the p.Gly2592 residue than for the p.Val1213 residue.

To further examine a possible significance of the TNXB variants, we performed immunostaining of a muscle biopsy obtained from the compound heterozygous proband. We found a strong reduction of tenascin-X in the ECM directly adjacent to the sarcolemma of skeletal muscle fibers of the patient compared to a healthy control (Fig. 4).

Furthermore, we investigated whether the COL1A1 splice mutation impairs type 1 collagen secretion. Using an ELISA, we found a normal secretion of type 1 procollagen by the fibroblasts from the patient's mother, but slightly reduced type 1 procollagen secretion in the index patient (Fig. 4B).

\section{DISCUSSION}

The patient presented here showed a complex phenotype including severe muscular weakness, joint hypermobility and bone fragility. First, he was considered to suffer from a congenital myopathy or neuropathy. However, muscle biopsy or respective genetic analyses were normal. To find the cause of this unclassifiable disease and investigate it on a more global level, WES was performed. This analysis revealed a mutation in COL1A1 also carried by the patient's mother as well as compound heterozygous variants in TNXB.

According to molecular mechanisms of the disease, two types of OI can be distinguished. In normally formed connective tissue, two $\alpha 1$ and one $\alpha 2$ polypeptide chain intertwine into a triple helix maintained by glycine residues at regular intervals to form collagen type 1 [Michou and Brown, 2011]. Due to a dominant negative 

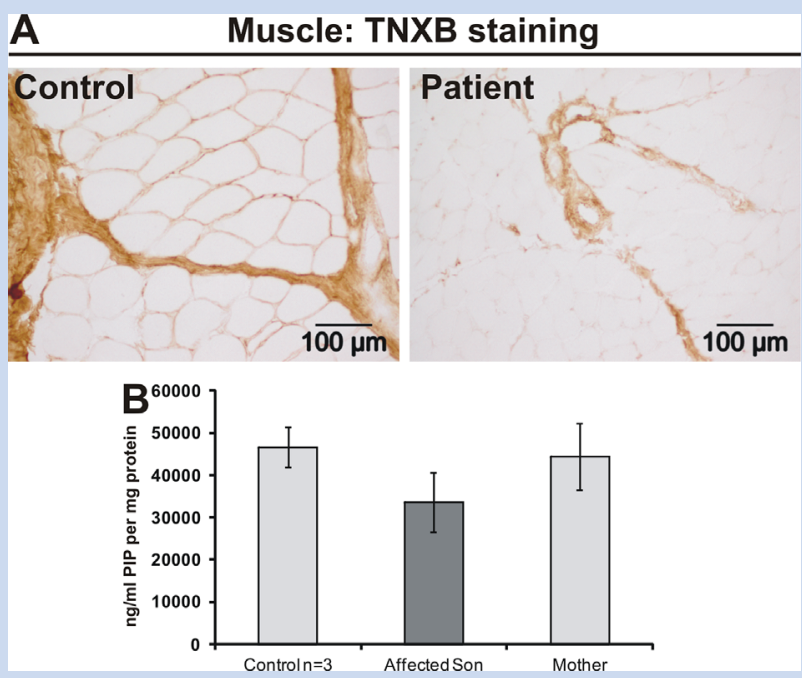

FIG. 4. Investigations of the effects of the TNXB variants and the COL1A1 mutation: [A] shows immunohistochemical staining of muscle with an anti-TNXB antibody. TNXB is strongly expressed in the extracellular matrix adjacent to the sarcolemma of skeletal muscle fibers of our age-matched control patient, but lacks almost completely in our index patient. This might lead to the decreased production of COL1A1 propeptide of the patients fibroblasts, as shown in $(B)$, whereas the mother with the monoallelic variant of TNXB has normal amount of COL1A1. [Color figure can be seen in the online version of this article, available at http://wileyonlinelibrary.com/journal/ajmga].

effect, mutations resulting in a protein still capable of forming the triple helix cause a more severe phenotype than mutations that lead to decreased collagen production and thus, chain exclusion [Basel and Steiner, 2009]. As expected by the position of the COL1A1 mutation, which changes the splice acceptor site of intron 49 from AG to AA, we detected retention of intron 49 in cDNA analysis of the patient.

Marini et al. [2007] reported 102 splice site mutations in the core exons of COL1A1 with only 10 of them leading to a lethal phenotype. This suggests that most splice site mutations in COL1A1 do not lead to a severe phenotype, possibly because: (i) the correct splice product might still be produced to some degree; and (ii) the abnormal product is likely to undergo nonsense-mediated decay. The first case of a C-terminal splice site mutation in COL1A1 was reported by Symoens et al. [2004] in a patient who also showed a mild overlapping OI/EDS phenotype. Overall, the mild phenotype presented by Symoens et al. correlates very well to the phenotype in our patient's mother, which was only identified as an OI phenotype when investigated very thoroughly. The normal secretion of COL1A1 in fibroblasts from the mildly affected mother suggests that the mutated transcript might be translated. Furthermore, it was possible to detect the mutated transcript without mRNA stabilizing agents. Both observations indicate that the presence of a mutated translation product may play a role in the pathogenesis. The mutated gene product could be partially functional, thereby explaining that C-terminal splice site mutations are associated with mild phenotypes. On the other hand, the mutated gene product could interfere with the collagen quaternary structure in a dominant negative manner.

A possible explanation for the more pronounced phenotype in the child might also be maternal mosaicism. Although Sanger sequencing of a maternal blood sample showed no sign of mosaicism, this cannot be completely ruled out since no other tissue has been investigated. Another option would be a difference in the ratio between mRNA expression from the healthy and the mutated allele in the mother and the index patient. In addition, other genetic factors might have an impact on the child's phenotype, especially on the additional severe muscle weakness. Our results indicate that the compound heterozygous state for both TNXB variants or only the heterozygous state for the p.Gly2592Ser variant are associated with TNXB deficiency found in his muscle tissue which may be an explanation for the more severe phenotype compared to the mother.

Most of the biallelic mutations in TNXB known to cause hypermobility EDS phenotype are truncating mutations (i.e., deletions, stop, or frameshift mutations) [Zweers et al., 2003]. However, Zweers et al. [2005] demonstrated that also biallelic missense mutations can cause an EDS-phenotype.

It is well known that EDS due to TNXBdeficiency usually presents with significant muscular hypotonia as seen in our patient. In addition, Pénisson-Besnier et al. [2013] presented a patient in his fourth decade with compound heterozygous mutations in TNXB leading to the phenotype of primary myopathy. It has been proposed that these muscular symptoms are due to the effect on the expression and fibril formation of type 4 collagen known to be associated with muscular dysfunction such as in Bethlem myopathy or Ullrich congenital muscular dystrophy [Kihara and Igarashi, 2004; Minamitani et al., 2004]. Like in the case presented here, immunostaining confirmed significantly less tenascin- $\mathrm{X}$ in muscle tissue in these patients [De Paepe and Malfait, 2012].

The close biochemical interaction between collagen type 1 fibres and tenascin- $\mathrm{X}$ is highlighted by investigations in $\operatorname{Tn} x b^{-/-}$mice: While the skin was histologically normal its collagen content was significantly reduced and $T n x b^{-1-}$ fibroblasts failed to deposit type 1 collagen into cell-associated matrix in vitro [Mao et al., 2002]. On the other hand, the two tenascin-X missense variants described here are unlikely to lead to a complete loss of the protein, but will rather change its function. As indicated by immunostaining, the deposition of mutant protein into the ECM appears to be hampered, which alone will dramatically alter its function. One important function of tenascin-X seems to be the regulation of TGF- $\beta$ activity in concert with myriads of other ECM proteins, among them also collagen type 1 [Alcaraz et al., 2014]. Therefore, tenascin-X alterations might lead to reduced production of collagen type 1 as observed in our in vitro experiment, thus creating a vicious circle, similar to other connective tissue disorders. Most connective tissue disorders, even the prototypical Marfan syndrome, are characterized by low bone mineral density [Noordam et al., 2009; Grover et al., 2012]. While certainly mechanical factors may play a role (lax ligaments, less muscle force), TGF- $\beta$ alterations might be a common culprit as indicated in a Marfan mouse model [Smaldone et al., 2015].

Our results show that individually mild genetic alterations in two extracellular matrix proteins, type 1 collagen and tenascin-X, 
may lead to a severe phenotype when they are combined. In this particular case, the COL1A1 mutation in the patient and the additional TNXB variants lead to a combined skeletal/connective tissue disease including muscular, joint, and bone problems. This case report is an example on how comprehensive genotyping by next-generation sequencing can help to elucidate the basis for complex phenotypes.

\section{ACKNOWLEDGMENTS}

We thank the patient and his parents for participating. Petra Matylewsky is gratefully acknowledged for expert technical assistance.

\section{REFERENCES}

Alcaraz LB, Exposito JY, Chuvin N, Pommier RM, Cluzel C, Martel S, Sentis S, Bartholin L, Lethias C, Valcourt U. 2014. Tenascin-X promotes epithelial-to-mesenchymal transition by activating latent TGF-beta. J Cell Biol 205:409-428.

Basel D, Steiner RD. 2009. Osteogenesis imperfecta: Recent findings shed new light on this once well-understood condition. Genet Med 11:375-385.

Beighton P, De Paepe A, Steinmann B, Tsipouras P, Wenstrup RJ. 1998. Ehlers-Danlos syndromes: Revised nosology, Villefranche, 1997. EhlersDanlos National Foundation (USA) and Ehlers-Danlos Support Group (UK). Am J Med Genet 77:31-37.

Cole WG. 1993. Etiology and pathogenesis of heritable connective tissue diseases. J Pediatr Orthop 13:392-403.

De Paepe A, Malfait F. 2012. The Ehlers-Danlos syndrome, a disorder with many faces. Clin Genet 82:1-11.

Grahame R. 1999. Joint hypermobility and genetic collagen disorders: Are they related? Arch Dis Child 80:188-191.

Grover M, Brunetti-Pierri N, Belmont J, Phan K, Tran A, Shypailo RJ, Ellis KJ, Lee BH. 2012. Assessment of bone mineral status in children with Marfan syndrome. Am J Med Genet A 158A:2221-2224.

Heinrich V, Kamphans T, Stange J, Parkhomchuk D, Hecht J, Dickhaus T, Robinson PN, Krawitz PM. 2013. Estimating exome genotyping accuracy by comparing to data from large scale sequencing projects. Genome Med 5:69.

Kamphans T, Krawitz PM. 2012. GeneTalk: An expert exchange platform for assessing rare sequence variants in personal genomes. Bioinformatics 28:2515-2516.

Kihara A, Igarashi Y. 2004. Cross talk between sphingolipids and glycerophospholipids in the establishment of plasma membrane asymmetry. Mol Biol Cell 15:4949-4959.

Li H. 2011. A statistical framework for SNP calling, mutation discovery, association mapping, and population genetical parameter estimation from sequencing data. Bioinformatics 27:2987-2993.

Mao JR, Taylor G, Dean WB, Wagner DR, Afzal V, Lotz JC, Rubin EM, Bristow J. 2002. Tenascin-X deficiency mimics Ehlers-Danlos syndrome in mice through alteration of collagen deposition. Nat Genet 30:421-425.

Marini JC, Blissett AR. 2013. New genes in bone development: What's new in osteogenesis imperfecta. J Clin Endocrinol Metab 98:3095-3103.

Marini JC, Forlino A, Cabral WA, Barnes AM, San Antonio JD, Milgrom S, Hyland JC, Korkko J, Prockop DJ, De Paepe A, Coucke P, Symoens S, Glorieux FH, Roughley PJ, Lund AM, Kuurila-Svahn K, Hartikka H, Cohn DH, Krakow D, Mottes M, Schwarze U, Chen D, Yang K, Kuslich
C, Troendle J, Dalgleish R, Byers PH. 2007. Consortium for osteogenesis imperfecta mutations in the helical domain of type I collagen: Regions rich in lethal mutations align with collagen binding sites for integrins and proteoglycans. Hum Mutat 28:209-221.

Michou L, Brown JP. 2011. Genetics of bone diseases: Paget's disease, fibrous dysplasia, osteopetrosis, and osteogenesis imperfecta. Joint Bone Spine 78:252-258.

Minamitani T, Ariga H, Matsumoto K. 2004. Deficiency of tenascin-X causes a decrease in the level of expression of type VI collagen. Exp Cell Res 297:49-60.

Noordam C, Funke S, Knoers NV, Jira P, Wevers RA, Urban Z, Morava E. 2009. Decreased bone density and treatment in patients with autosomal recessive cutis laxa. Acta Paediatr 98:490-494.

Penisson-Besnier I, Allamand V, Beurrier P, Martin L, Schalkwijk J, van Vlijmen-Willems I, Gartioux C, Malfait F, Syx D, Macchi L, Marcorelles P, Arbeille B, Croue A, De Paepe A, Dubas F. 2013. Compound heterozygous mutations of the TNXB gene cause primary myopathy. Neuromuscul Disord 23:664-669.

Preusse C, Goebel HH, Held J, Wengert O, Scheibe F, Irlbacher K, Koch A, Heppner FL, Stenzel W. 2012. Immune-mediated necrotizing myopathy is characterized by a specific Th1-M1 polarized immune profile. Am J Pathol 181:2161-2171.

Shapiro JR, Lietman C, Grover M, Lu JT, Nagamani SC, Dawson BC, Baldridge DM, Bainbridge MN, Cohn DH, Blazo M, Roberts TT, Brennen FS, Wu Y, Gibbs RA, Melvin P, Campeau PM, Lee BH. 2013. Phenotypic variability of osteogenesis imperfecta type V caused by an IFITM5 mutation. J Bone Miner Res 28:1523-1530.

Smaldone S, Clayton NP, Del Solar M, Pascual-Gonzales G, Cheng SH, Wentworth BM, Schaffler MB, Ramirez F. 2015. Fibrillin-1 regulates skeletal stem cell differentiation by modulating TGFbeta activity within the marrow niche. J Bone Miner Res. doi 10.1002/jbmr2598 [Epub ahead of print]

Symoens S, Nuytinck L, Legius E, Malfait F, Coucke PJ, De Paepe A. 2004. Met $>$ Val substitution in a highly conserved region of the pro-alpha1(I) collagen C-propeptide domain causes alternative splicing and a mild EDS/OI phenotype. J Med Genet 41:e96.

Van Dijk FS, Sillence DO. 2014. Osteogenesis imperfecta: Clinical diagnosis, nomenclature, and severity assessment. Am J Med Genet A 164A:1470-1481.

Voermans NC, Altenburg TM, Hamel BC, de Haan A, van Engelen BG. 2007. Reduced quantitative muscle function in tenascin-X deficient Ehlers-Danlos patients. Neuromuscul Disord 17:597-602.

Voermans NC, Bonnemann CG, Huijing PA, Hamel BC, van Kuppevelt TH, de Haan A, Schalkwijk J, van Engelen BG, Jenniskens GJ. 2008. Clinical and molecular overlap between myopathies and inherited connective tissue diseases. Neuromuscul Disord 18:843-856.

Wang K, Li M, Hakonarson H. 2010. ANNOVAR: Functional annotation of genetic variants from high-throughput sequencing data. Nucleic Acids Res 38:e164.

Zweers MC, Bristow J, Steijlen PM, Dean WB, Hamel BC, Otero M, Kucharekova M, Boezeman JB, Schalkwijk J. 2003. Haploinsufficiency of TNXB is associated with hypermobility type of Ehlers-Danlos syndrome. Am J Hum Genet 73:214-217.

Zweers MC, Dean WB, van Kuppevelt TH, Bristow J, Schalkwijk J. 2005. Elastic fiber abnormalities in hypermobility type Ehlers-Danlos syndrome patients with tenascin-X mutations. Clin Genet 67:330-334.

\section{SUPPORTING INFORMATION}

Additional supporting information may be found in the online version of this article at the publisher's web-site. 